\title{
Percutaneous Transhepatic Laser Lithotripsy for Biliary Stones in Patients with Altered Biliary Anatomy
}

\author{
Jacob Indu' ${ }^{1, \odot ~ V i k r a m a ~ A m i t h a ~ K h e d a ~}{ }^{1}$ Deepak Bolbandi ${ }^{2}$ Sanjay Govil ${ }^{3}$ Ravisankar Bhat ${ }^{4}$
}

${ }^{1}$ Department of Interventional Radiology, Apollo Hospital, Bangalore, Karnataka, India

${ }^{2}$ Department of Urology, Apollo Hospital, Bangalore, Karnataka, India

${ }^{3}$ Department of Gastrointestinal Surgery and Liver Transplantation, Apollo Hospital, Bangalore, Karnataka, India

${ }^{4}$ Department of Gastrointestinal Surgery, Apollo Hospital, Bangalore, Karnataka, India
Address for correspondence Indu Jacob, MD, DNB, Department of Interventional Radiology, Apollo Hospital, Bangalore, Karnataka, India (e-mail: indualfred@gmail.com).

\begin{abstract}
Keywords

- percutaneous lithotripsy

- biliary stones

- holmium laser

Endoscopic retrograde cholangiopancreatography (ERCP) is the current treatment of choice in bile duct stones. Several factors such as variant anatomy of ampulla and surgical procedures like hepaticojejunostomy limit the success of ERCP in treating bile duct stones. Percutaneous transhepatic laser lithotripsy using interventional radiologic and endourologic techniques, which is uncommon, is a reasonable treatment option in such difficult cases. It is a minimally invasive, safe procedure accompanied by a high success rate, minimal morbidity, and a short hospital stay. We report our technique and experience in a series of three patients who underwent percutaneous transhepatic biliary drainage (PTBD) followed by percutaneous transhepatic laser lithotripsy in an attempt to avoid open surgery when ERCP was technically difficult.
\end{abstract}

\section{Introduction}

Currently, the treatment of choice in bile duct stones is endoscopic retrograde cholangiopancreatography (ERCP), followed by laparoscopic cholecystectomy. ${ }^{1}$ However, factors such as variant anatomy of the ampulla of Vater, presence of duodenal diverticula, surgical procedures such as roux en $\mathrm{Y}$ gastric bypass, and hepaticojejunostomy can limit the success of ERCP. Options in such cases are limited and include peritoneoscopic biliary surgery, transhepatic stone removal using dormia basket, or predilating the papilla with balloons and clearing the stones. ${ }^{2}$ Laser lithotripsy, by either per-oral endoscopy or a percutaneous transhepatic approach, is a very good alternative for such difficult/complicated biliary stones. We report our technique and experience in a series of three patients who underwent percutaneous transhepatic biliary drainage (PTBD), followed by percutaneous transhepatic laser lithotripsy in an attempt to avoid open surgery when ERCP was technically difficult.

\section{Case 1}

A 49-year-old female, who underwent Whipple surgery for pancreatic carcinoma 5 years ago, presented with fever, vomiting, and pruritus. Laboratory values showed signs of cholestasis and inflammation (-Table $\mathbf{1}$ ).
DOI https://doi.org/

$10.1055 / \mathrm{s}-0041-1728985$

ISSN 2457-0214 (c) 2021. Indian Society of Vascular and Interventional Radiology. This is an open access article published by Thieme under the terms of the Creative Commons Attribution-NonDerivative-NonCommercial-License, permitting copying and reproduction so long as the original work is given appropriate credit. Contents may not be used for commercial purposes, or adapted, remixed, transformed or built upon. (https://creativecommons.org/licenses/by-nc-nd/4.0/). Thieme Medical and Scientific Publishers Pvt. Ltd. A-12, 2nd Floor, Sector 2, Noida-201301 UP, India 
Table 1 Table showing improvement in LFT values postprocedure

\begin{tabular}{|l|l|l|l|}
\hline Cases & Criteria & Preoperative & Postoperative \\
\hline \multirow{5}{*}{ Case 1} & ALP & $762 \mathrm{U} / \mathrm{L}$ & $215 \mathrm{U} / \mathrm{L}$ \\
\cline { 2 - 4 } & GGT & $666 \mathrm{U} / \mathrm{L}$ & $78 \mathrm{U} / \mathrm{L}$ \\
\cline { 2 - 4 } & D. bilirubin & $1.89 \mathrm{mg} / \mathrm{dl}$ & $0.13 \mathrm{mg} / \mathrm{dl}$ \\
\cline { 2 - 4 } & TC & 13500 & 9000 \\
\cline { 2 - 4 } & Neutrophils 2 & $84 \%$ & $40-80 \%$ \\
\hline \multirow{5}{*}{ Case } & ALP & $165 \mathrm{U} / \mathrm{L}$ & $121 \mathrm{U} / \mathrm{L}$ \\
\cline { 2 - 4 } & GGT & $180 \mathrm{U} / \mathrm{L}$ & $51 \mathrm{U} / \mathrm{L}$ \\
\cline { 2 - 4 } & D. bilirubin & $2.28 \mathrm{mg} / \mathrm{dl}$ & $0.19 \mathrm{mg} / \mathrm{dl}$ \\
\cline { 2 - 4 } & TC & 13000 & 8500 \\
\cline { 2 - 4 } & Neutrophils & $90 \%$ & $40-80 \%$ \\
\hline & ALP & $977 \mathrm{U} / \mathrm{L}$ & $290 \mathrm{U} / \mathrm{L}$ \\
\cline { 2 - 4 } & GGT & $1232 \mathrm{U} / \mathrm{L}$ & $141 \mathrm{U} / \mathrm{L}$ \\
\cline { 2 - 4 } & D. bilirubin & $0.23 \mathrm{mg} / \mathrm{dl}$ & $0.14 \mathrm{mg} / \mathrm{dl}$ \\
\cline { 2 - 4 } & TC & 16000 & 9000 \\
\cline { 2 - 4 } & Neutrophils & $90 \%$ & $40-80 \%$ \\
\hline
\end{tabular}

Abbreviations: ALP, alkaline phosphatase; D. bilirubin, direct bilirubin; GGT, gamma glutamyl transferase; LFT, liver function test; TC, total count.

Magnetic resonance cholangiopancreatography (MRCP) showed common hepatic duct (CHD) stone of size $13 \times 24 \times$ $42 \mathrm{~mm}$ close to hepaticojejunostomy $(\mathrm{HJ})$ site with mild biliary dilatation and possible stricture at the anastomosis ( - Fig. 1A). Hence, the final diagnosis was choledocholithiasis and cholangitis. As ERCP was difficult due to post HJ status, PTBD followed by interval percutaneous transhepatic laser lithotripsy was planned.

- Percutaneous transhepatic biliary drainage: Broadspectrum antibiotic (inj. Cefoperazone $1000 \mathrm{mg}+500 \mathrm{mg}$ sulbactam) was given 1 hour before the procedure and 8 hours after the procedure. The procedure was done under local anesthesia and IV sedation. Under ultrasound and fluoroscopic guidance, the left hepatic duct branch was punctured, and access was secured by $5 \mathrm{~F}$ sheath (Accustick; Boston Scientific), followed by $7 \mathrm{~F}$ sheath (Translumina 7F femoral introducer sheath). A $5 \mathrm{~F}$ catheter (40 cm Kumpe catheter) was navigated into the left hepatic duct, and cholangiogram showed intrahepatic biliary radical dilatation (IHBRD) and a large filling defect in the CHD close to $\mathrm{HJ}$ site suggestive of calculus. A hydrophilic guidewire (260 cm 035" angled tip; Terumo) was negotiated across the CHD stone into the jejunum ( $\mathbf{- F i g . ~ 1 B ) , ~ w h i c h ~}$ was exchanged for a stiff guidewire ( $260 \mathrm{~cm} 035$ "J tip PTFE coated; Translumina). An $8.5 \mathrm{~F}$ internal-external drainage catheter was placed across the stone into the jejunum (-Fig. 1C). The postoperative period was uneventful and she was discharged with PTBD catheter closed externally. Three weeks later, she got admitted for percutaneous laser lithotripsy.

- Percutaneous transhepatic laser lithotripsy: Procedure was done under general anesthesia (GA) with antibiotic prophylaxis. Under fluoroscopic guidance, contrast was injected into the PTBD catheter, which showed the contrast in the jejunum. The PTBD catheter was removed over a stiff guidewire (035" $260 \mathrm{~cm}$ J tip PTFE coated; Translumina). The PTBD tract was dilated with tissue dilators, and a $12 \mathrm{~F}$ access sheath (flexor ureteral access sheath with AQ hydrophilic coating; $12 \mathrm{~F} / 35 \mathrm{~cm}$; Cook Medical) was inserted (-Fig. 2A). Through the 12 Fr sheath, a flexible choledochoscope (KARL STORZ 11292 ADU1) was introduced for direct visualization of stones (-Fig. 2B). Laser lithotripsy of the stone was

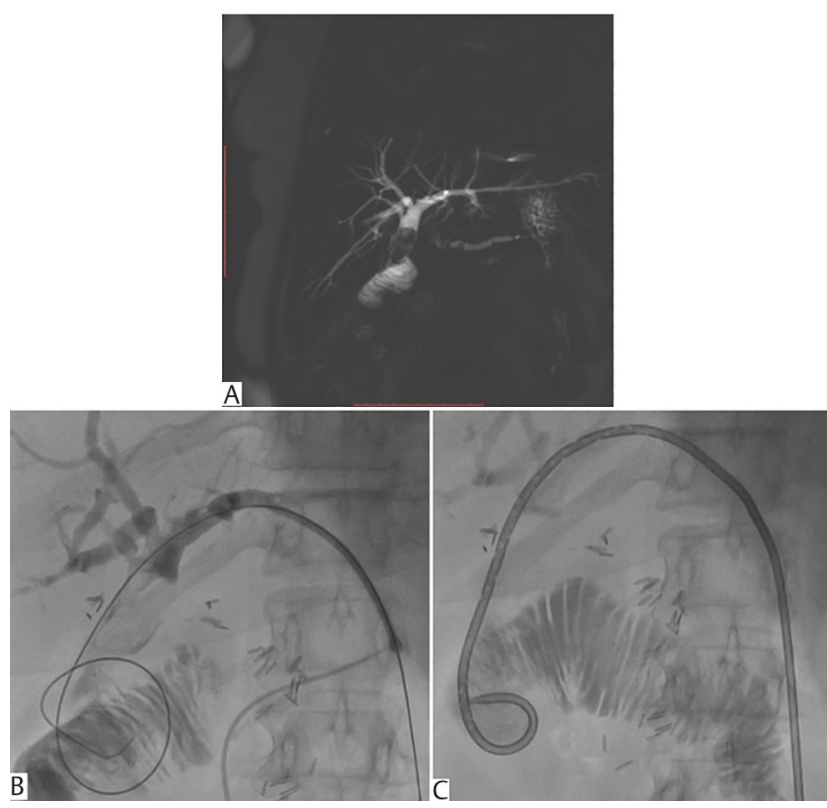

Fig. 1 (A) Case 1-Magnetic resonance cholangiopancreatography (MRCP) showing large stone in the common hepatic duct with mild biliary dilatation and possible stricture at the hepaticojejunostomy (HJ) anastomosis and pancreatic ductal calculi can also be seen. (B) Case 1-Percutaneous transhepatic cholangiogram showing a large filling defect near the $\mathrm{HJ}$ site suggestive of calculus. (C) Case 1-Fluoroscopy image showing an $8.5 \mathrm{~F}$ internal-external percutaneous transhepatic biliary drainage (PTBD) catheter across the calculus. 
done using holmium laser fiber (Lumenis slimline SIS 365 micron). Continuous warm saline irrigation was done during lithotripsy. After lithotripsy, choledochoscope was removed over the guidewire, and balloon plasty of the CHD and hepaticojejunostomy site was done using a $10 \times 40 \mathrm{~mm}$ balloon catheter ( $\mathbf{- F i g}$. 2C). The final cholangiogram obtained through a $10 \mathrm{~F}$ vascular sheath showed adequate drainage of bile without filling defects (-Fig. 2D). A $14 \mathrm{~F}$ internal-external drainage catheter was placed across the $\mathrm{HJ}$ into the jejunum (-Fig. 2E). The patient tolerated the procedure well, and she was discharged in a stable condition with the PTBD catheter closed externally (-Table 1). Follow-up cholangiogram after 3 months showed free flow of contrast into the jejunum with no filling defects/IHBRD ( $\mathbf{- F i g . ~ 2 F ) . ~ H e n c e , ~ t h e ~}$ PTBD catheter was removed.

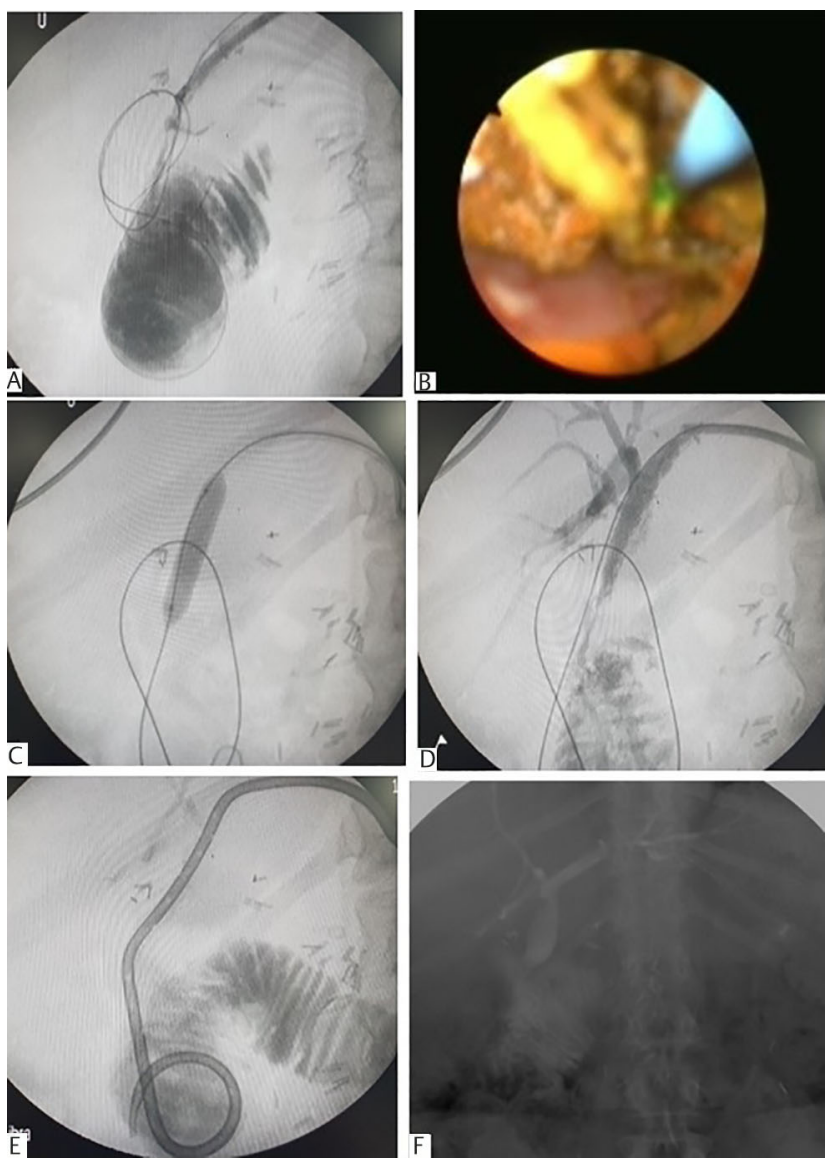

Fig. 2 (A) Case 1-Fluoroscopy image showing $12 \mathrm{~F}$ sheath for cholangioscopy which was exchanged with percutaneous transhepatic biliary drainage (PTBD) catheter over an 035 guidewire after tract dilatation. (B) Case 1-Cholangioscopy image showing stone within the common hepatic duct (CHD). (C) Case 1-Fluoroscopy image showing balloon dilatation of the hepaticojejunostomy $(\mathrm{HJ})$ site done with a $10 \times 40 \mathrm{~mm}$ balloon catheter after LASER lithotripsy. (D) Case 1-Cholangiogram showing the free flow of bile after LASER lithotripsy and balloon plasty of the HJ site. (E) Case 1-Fluoroscopy image showing $14 \mathrm{~F}$ internal-external drainage catheter placed across the $\mathrm{HJ}$ after lithotripsy and balloon plasty of $\mathrm{HJ}$ site. (F) Case 1-Follow-up cholangiogram demonstrated adequate drainage of bile without any filling defects.

\section{Case 2}

A 72-year-old male presented with abdominal pain, fever, and vomiting. His laboratory values showed signs of cholestasis and inflammation (Table 1). USG abdomen showed features of acute calculous cholecystitis. MRCP showed choledocholithiasis and calculus cholecystitis. The final diagnosis was acute calculous cholecystitis, choledocholithiasis, and cholangitis. ERCP was difficult as there was a history of roux-en-Y gastrojejunostomy for duodenal ulcer. Hence, after all preoperative evaluation, he underwent laparotomy, adhesiolysis, subtotal cholecystostomy, exploration of common bile duct (CBD), choledocholithotomy, intraoperative cholangiogram, and T- tube placement, under GA. The postoperative period was uneventful and he was discharged in a stable condition. One month later, he started complaining of right upper quadrant pain. T-tube cholangiogram showed IHBRD and two filling defects of size $9 \times 13 \mathrm{~mm}$ and $10 \times 14 \mathrm{~mm}$ suggestive of CBD calculi ( - Fig. 3A). As ERCP was difficult, he underwent PTBD ( - Fig. 3B, C), followed by interval laser lithotripsy for the clearance of $\mathrm{CBD}$ calculi. The patient was discharged in a stable condition with the PTBD catheter closed externally (-Table 1). Follow-up cholangiogram after 2 weeks showed adequate drainage of bile with no filling defects. Hence, the PTBD catheter was removed.

\section{Case 3}

A 75-year-old male, who underwent hepaticojejunostomy for choledochal cyst 10 years ago, presented with fever for 1 month. His laboratory values showed signs of cholestasis
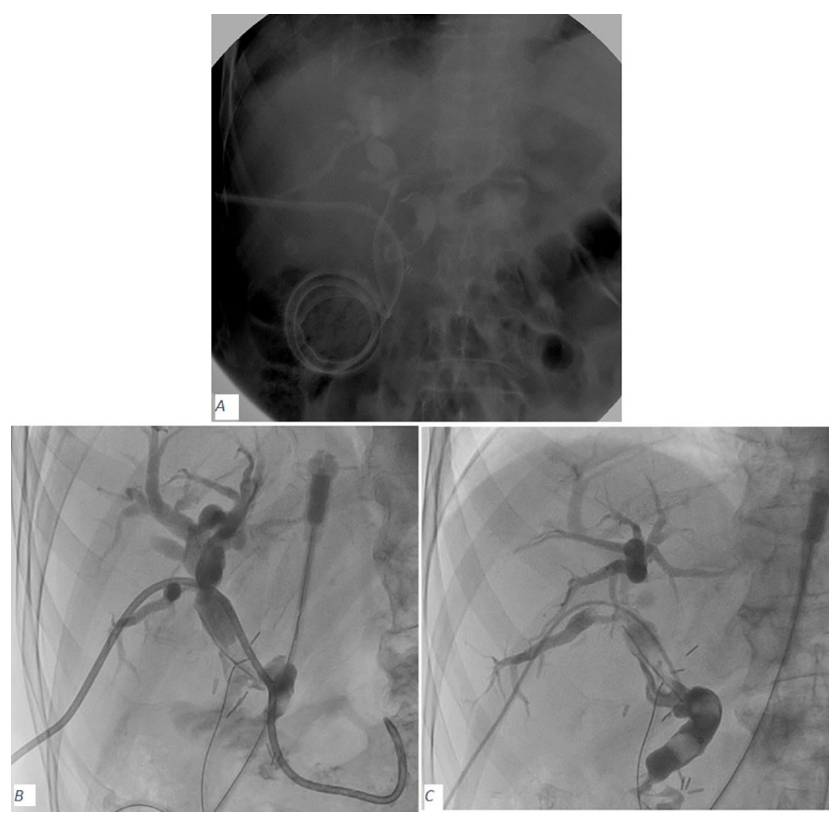

Fig. 3 (A) Case 2-T tube cholangiogram showing filling defects in the common bile duct (CBD) suggestive of calculus. (B) Case 2-Percutaneous transhepatic cholangiogram showing filling defects in the CBD suggestive of stone. (C) Case 2-Fluoroscopy image showing an $8.5 \mathrm{~F}$ percutaneous transhepatic biliary drainage (PTBD) catheter placed across the CBD stone into the duodenum. 
and inflammation (-Table 1). Ultrasound abdomen showed IHBRD with a calculus of size $20 \times 17 \times 28 \mathrm{~mm}$ in the CHD near the $\mathrm{HJ}$ site. The final clinical diagnosis was posthepaticojejunostomy, obstructive jaundice due to CHD stone and cholangitis. As ERCP was difficult due to post HJ status, he underwent PTBD ( - Fig. 4A-C), followed by interval percutaneous transhepatic laser lithotripsy. Postlithotripsy, the patient developed fever and chills, and antibiotics were hiked as per blood culture report. He was discharged in a hemodynamically stable condition with the PTBD catheter closed externally (-Table 1). Follow-up cholangiogram at 3 months showed free flow of contrast into the jejunum with no filling defects/IHBRD ( - Fig. 4D,E). Hence, the PTBD catheter was removed.

\section{Discussion}

Bile duct stones occur as a result of either formation of stones within intrahepatic/extrahepatic bile ducts or the passage of gall stones into the CBD. CBD stones are estimated to be present in 10 to $20 \%$ of individuals with symptomatic gallstones. ${ }^{3}$ Bile duct stones are considered a troublesome postoperative adverse event in patients with a hepatobiliary pancreatic disease undergoing hepaticojejunostomy. These are mostly caused by cholestasis or reflux cholangitis. Both complete stone clearance and maintained resolution of any
$\mathrm{HJ} /$ intrahepatic stricture using minimally invasive treatment is ideal and essential for these patients. ${ }^{4}$

Currently, most of the biliary calculi are successfully removed either at the time of laparoscopic cholecystectomy or pre- or postoperatively with ERCP. Increasing populations of patients are unable to have their stones removed endoscopically because the stones are too large or impacted or previous surgical procedures make the papilla inaccessible. Open CBD exploration is reserved for such patients who are not candidates for endoscopic or laparoscopic procedures. However, it may be associated with other morbidities. Percutaneous stone removal is a minimally invasive technique for the management of such difficult cases. ${ }^{5}$

Percutaneous approach to biliary stone removal was first undertaken in 1962 when Dr. Mondet extracted a retained CBD stone through a $\mathrm{T}$ tube using forceps. Percutaneous access can be achieved by a variety of methods, namely, transhepatic, T-tube, cholecystostomy tube and, rarely, transcystic route. Transhepatic access is the most common route used nowadays. Various percutaneous techniques of biliary stone removal have been described in the literature, including stone extraction by forceps or baskets; antegrade expulsion of stones into the duodenum by forceful irrigation, or with the aid of various angioplasty balloon catheters, with or without stone fragmentation (lithotripsy); chemolitholysis; sphincteroplasty and stricture dilatation. ${ }^{6}$ Adapted from

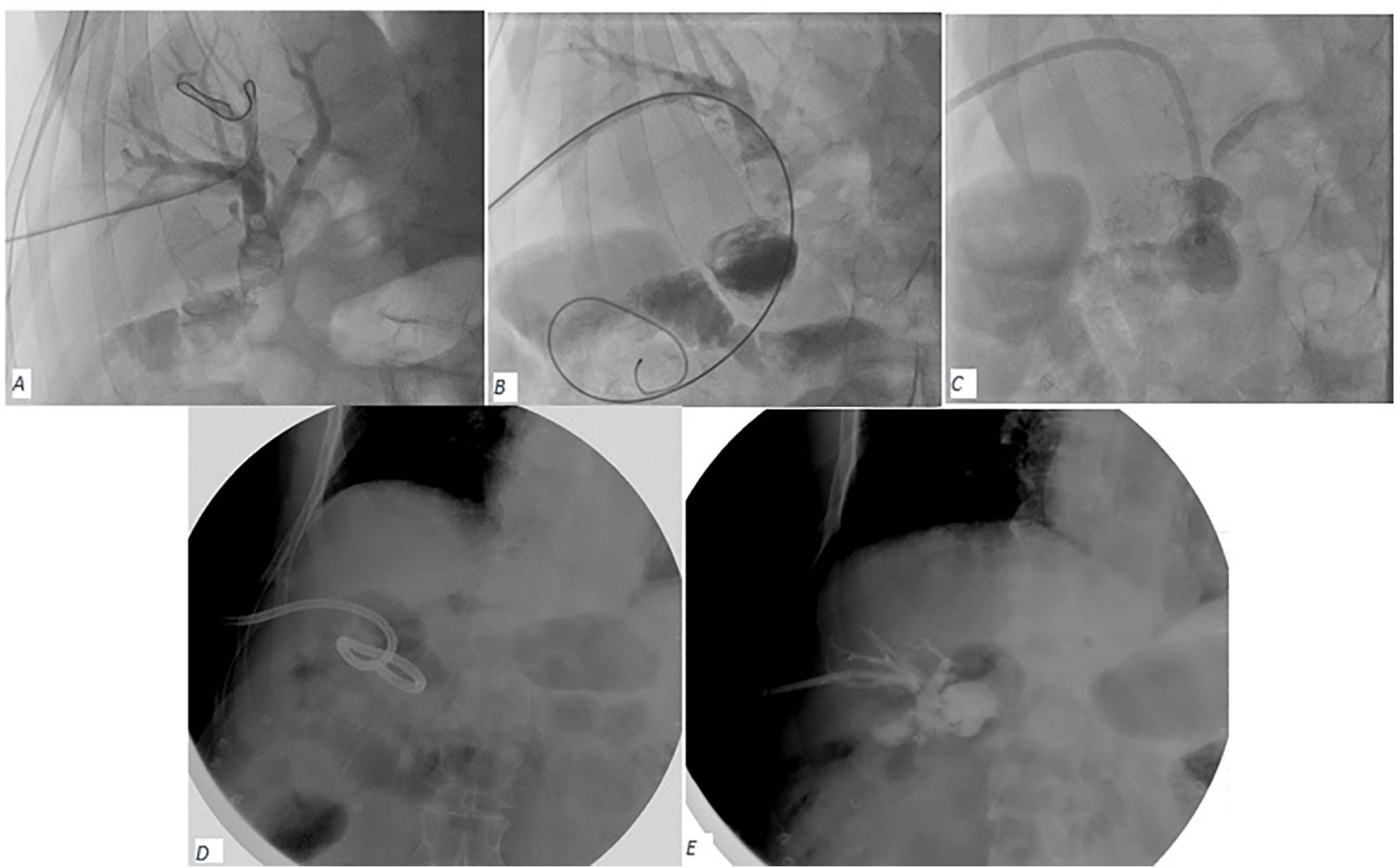

Fig. 4 (A) Case 3-Cholangiogram showing a large filling defect in the common hepatic duct (CHD) near the hepaticojejunostomy (HJ) site suggestive of calculus. (B) Case 3-Fluoroscopy image showing guidewire across the HJ stone into the jejunum. (C) Case 3-Fluoroscopy image showing a $7 \mathrm{~F}$ catheter placed across the $\mathrm{HJ}$ stone into the jejunum. (D) Case 3-Fluoroscopy image showing $14 \mathrm{~F}$ percutaneous transhepatic biliary drainage (PTBD) catheter (placed after lithotripsy) in situ. (E) Case 3-Follow-up cholangiogram showing the free flow of contrast into jejunum without any filling defects in the CHD. 
endoscopic management of urinary calculi, cholangioscopy has since emerged as an alternative method of stone clearance for complex cases of choledocholithiasis. Lithotripsy is a therapeutic adjunct that improves success rate in patients undergoing video cholangioscopy-guided clearance of biliary calculi, especially for the clearance of large, impacted, and irregularly shaped calculi. Lithotripsy may be performed by intracorporeal approaches using mechanical, electrohydraulic, or laser devices at the time of ERCP or percutaneous access, or by extracorporeal shock wave lithotripsy. Lee et al retrospectively reviewed 34 consecutive patients who underwent unsuccessful removal of CBD stones using conventional endoscopic methods and were subsequently treated using percutaneous cholangioscopy-guided stone clearance (combining conventional methods and electrohydraulic lithotripsy). Complete stone clearance was achieved in all cases with only $6 \%$ major complication rate (hemobilia, PTBD tract disruption). ${ }^{7}$

The holmium laser is a small contact lithotrite (200 $\mu \mathrm{m}$ to $1000 \mu \mathrm{m}$ ) that effectively fragments urinary stones with minimal stone retropulsion and subsequent collateral damage to surrounding tissue. Percutaneous transhepatic cholangioscopic laser lithotripsy with holmium:yttrium-aluminum-garnet (holmium: YAG) laser has been shown to effectively clear stones without serious procedure-related complications. ${ }^{8}$

Several small series, with a mean of 16 patients (range 9-22 patients) have validated the usefulness of holmium laser lithotripsy for the treatment of difficult biliary calculi. Shamamian and Grasso reported a much larger series of 36 patients with complex intrahepatic biliary calculi (22 primary and 14 secondary intrahepatic calculi); of these, 22 were treated with percutaneous endoscopic holmium laser lithotripsy. Complete stone clearance was accomplished in all patients without major complications. No recurrence or major complications were identified during the follow-up period ranging from 6 to 25 months. ${ }^{9}$

Recently, percutaneous transhepatic cholangioscopy (PTCS) with Spyglass Direct visualization system has been described, which allows access to the biliary tree with direct visualization and sampling of the bile duct via cholangioscopy, and additionally facilitates optically guided intraductal fragmentation and clearance of biliary calculi. ${ }^{10}$

Tsutsumi et al have compared treatment methods for bile duct stones after hepaticojejunostomy between percutaneous transhepatic cholangioscopy (PTCS) and peroral, short double-balloon enteroscopy (sDBE). Forty consecutive patients were treated for bile duct stones after hepaticojejunostomy. The initial success rates for biliary access were $100 \%$ with PTCS and $91 \%$ with sDBE. The rate of true complete stone clearance was similar to PTCS and SDBE. He concluded that, compared with PTCS, peroral endoscopic procedures using an SDBE for managing bile duct stones in patients with prior hepaticojejunostomy was useful and had low rates of adverse events and a short hospitalization. ${ }^{4}$
Our series of patients had large/complex biliary calculi that were effectively managed through the coordinated efforts of the interventional radiology and urology teams. All three patients were visually stone-free after one cholangioscopic lithotripsy procedure. No major perioperative/postoperative complications occurred. The mean length of hospital stay was 2 days. At 3 months radiologic follow-up, no retained stones/stone recurrence was noted. Although previous reports of this technique exist, this case series contributes to the growing body of literature supporting the use of multidisciplinary techniques, including interventional radiology in treating retained and sometimes large biliary calculi.

In conclusion, percutaneous transhepatic laser lithotripsy in our experience represents a minimally invasive approach, safe, accompanied by a high-success rate, minimal morbidity, and a short hospital stay. It can be considered for those cases in which an endoscopic approach has failed or is technically difficult. Success depends on a multidisciplinary approach.

Conflict of Interest

None declared.

\section{References}

1 Williams E, Beckingham I, El Sayed G, et al. Updated guideline on the management of common bile duct stones (CBDS) Gut 2017;66(5):765-782

2 Gupta P, Gupta J, Praveen KM. Imaging in Obstructive Jaundice: What a Radiologist Needs to Know before Doing a Percutaneous Transhepatic Biliary Drainage. J Clin Interv Radiol ISVIR 2020;4(1):31-37

3 Tazuma S. Gallstone disease: Epidemiology, pathogenesis, and classification of biliary stones (common bile duct and intrahepatic). Best Pract Res Clin Gastroenterol 2006;20(6):1075-1083

4 Tsutsumi K, Kato H, Yabe S, et al. A comparative evaluation of treatment methods for bile duct stones after hepaticojejunostomy between percutaneous transhepatic cholangioscopy and peroral, short double-balloon enteroscopy. Therap Adv Gastroenterol 2017;10(1):54-67

5 Ierardi AM, Fontana F, Petrillo M, et al. Percutaneous transhepatic endoscopic holmium laser lithotripsy for intrahepatic and choledochal biliary stones. Int J Surg 2013;11(Suppl 1) :S36-S39

6 Cheng JHM, Leung WK, Wong AHC, et al. Percutaneous transhepatic biliary stones removal- An effective and safe alternative. Hong Kong J Radiol 2020;23:106-113

7 Lee JH, Kim HW, Kang DH, et al. Usefulness of percutaneous transhepatic cholangioscopic lithotomy for removal of difficult common bile duct stones. Clin Endosc 2013;46(1):65-70

8 Healy K, Chamsuddin A, Spivey J, Martin L, Nieh P, Ogan K. Percutaneous endoscopic holmium laser lithotripsy for management of complicated biliary calculi. JSLS 2009;13(2):184-189

9 Shamamian P, Grasso M. Management of complex biliary tract calculi with a holmium laser. J Gastrointest Surg 2004;8(2):191-199

10 Tripathi N, Mardini H, Koirala N, Raissi D, Emhmed Ali SM, Frandah WM. Assessing the utility, findings, and outcomes of percutaneous transhepatic cholangioscopy with Spyglass Direct visualization system: a case series. Transl Gastroenterol Hepatol 2020;5:12 